\title{
MARCADORES CUADRADOS Y DEFORMACIÓN DE OBJETOS EN NAVEGACIÓN QUIRÚRGICA CON REALIDAD AUMENTADA
}

\author{
Eliana Margarita Aguilar Larrarte \\ Universidad del Cauca, Popayán-Colombia, eaguilar@unicauca.edu.co \\ Oscar Andrés Vivas Albán \\ Universidad del Cauca, Popayán-Colombia, avivas@unicauca.edu.co \\ José María Sabater Navarro \\ Universidad Miguel Hernández, Elche-España, j.sabater@goumh.umh.es
}

\begin{abstract}
Resumen
Este artículo muestra el resultado de pruebas preliminares con librerías y entornos de desarrollo comerciales encaminados a la construcción de una aplicación para navegación quirúrgica en el campo de la laparoscopia, usando marcadores cuadrados, extensible a despliegue en dispositivos móviles y gafas de realidad virtual. Para la renderización de los objetos 3D se usó el Game Engine Unity junto a la librería Vuforia. Las pruebas preliminares muestran resultados satisfactorios en el seguimiento de marcadores y en la construcción de la información aumentada para ser usada por el cirujano con deformación física de objetos en tiempo real.
\end{abstract}

Palabras Clave: Realidad aumentada, cirugía laparoscópica, aplicaciones móviles, deformación de objetos.

\section{INTRODUCCIÓN}

La cirugía mínimamente invasiva es un tipo de procedimiento operatorio que se realiza con instrumental miniaturizado introducido a través de pequeñas incisiones. El cirujano efectúa el procedimiento manipulando los instrumentos, guiado principalmente por la imagen trasmitida gracias a una cámara endoscópica introducida por las pequeñas incisiones realizadas en el abdomen del paciente, la cual se encuentra equipada con una lámpara de luz fría [11].

La cirugía mínimamente invasiva ofrece importantes beneficios en la recuperación del paciente, disminuyendo el impacto físico de la intervención, pero conlleva importantes compromisos para los cirujanos debido a la miniaturización de los instrumentos y a las limitaciones visuales y táctiles, requiriendo gran experticia por parte de ellos [14]. Estas limitaciones se constituyen en una barrera como se evidencia en el estudio publicado en [15], en donde se muestran los resultados de una encuesta diseñada para examinar las barreras de adopción de la laparoscopia por ginecólogos en ejercicio. La encuesta se aplicó a 4.273 cirujanos ginecológicos a través de los Estados Unidos. De dicho estudio se puede abstraer que de los cirujanos que pueden aconsejar intervenciones mínimamente invasivas más de la mitad $(62.50 \%)$ no lo hace debido a las limitaciones visuales, y el $65.60 \%$ debido a la pérdida de visión binocular, convirtiéndose el aspecto visual en uno de los principales limitantes en la recomendación de una cirugía laparoscópica por parte de estos especialistas. Ante esta situación en los últimos años la tecnología ha incursionado en el campo quirúrgico mostrando un avance cada vez mayor, ya sea en el proceso de entrenamiento de cirujanos o asistiéndoles durante la intervención, creándose con el tiempo una buena variedad de sistemas de entrenamiento, asistencia y navegación. Para citar un caso, los sistemas de navegación quirúrgica construyen diagramas tridimensionales de datos médicos en tiempo real, ampliando la percepción visual, siendo de gran ayuda en los procesos de navegación quirúrgica [7], generando que las tecnologías computacionales tengan mayor futuro debido al aumento de la realidad y al acercamiento de ampliación con realidad mixta. En la última década las aplicaciones médicas de realidad aumentada ( $\mathrm{AR}$ ) han tenido una rápida expansión, originada principalmente por avances en el hardware (interfaces hápticas y despliegues), al mismo tiempo que los teléfonos inteligentes y tabletas se han constituido en herramientas cada vez más populares para aplicaciones en medicina, industria y educación [7].

El trabajo presentado en este artículo muestra un primer acercamiento a una aplicación móvil de realidad aumentada (MAR) para cirugía laparoscópica, usando la librería Vuforia para Unity, la cual permite desarrollar aplicaciones de realidad aumentada para Android o iOS, que sumado al motor de juego multiplataforma Unity permitió la construcción de una APK con seguimiento de marcadores cuadrados y deformación de objetos virtuales. 


\section{CIRUGÍA INVASIVA}

La cirugía laparoscópica al igual que otros tipos de cirugías mínimamente invasivas, trabaja bajo la disminución de las inserciones en el paciente usando instrumentos quirúrgicos especiales y un sistema de visión compuesto por una cámara y una lámpara de iluminación fría. Cámara y lámpara se conocen como endoscopio y junto con los otros instrumentos son insertados a través de pequeñas incisiones en la cavidad abdominal. La imagen del área de cirugía es desplegada en una pantalla y el cirujano es guiado principalmente por lo que ve en ella, manipulando los instrumentos y efectuando el proceso operatorio.

Este tipo de procedimiento quirúrgico evita cortes de gran extensión, pero debido a las características del método requiere alto nivel de experticia por parte del cirujano [8]. Muchos procedimientos quirúrgicos tradicionales han sido remplazados por esta técnica laparoscópica, dando origen a otras técnicas derivadas menos invasivas como es el caso de NOTES y LESS. Las cirugías NOTES (Natural Orifice Transluminal Endoscopic Surgery) se realizan a partir de un acceso a la cavidad abdominal a través de orificios naturales del cuerpo humano como la boca, la nariz, el ano y la vagina [1], [6]. En tanto que LESS (Laparo Endoscopic Single-Site Surgery) es una técnica donde dicho acceso se realiza por una única incisión [1], [11].

\section{REALIDAD AUMENTADA}

La realidad aumentada tiene como finalidad sobreponer imágenes tridimensionales sobre las imágenes proporcionadas por las cámaras [2]. Esta técnica tiene como uno de sus mayores retos minimizar la diferencia entre el mundo real y el objeto virtual, y aumentar la percepción de la realidad en el sentido de que el observador pueda ver la misma escena. La realidad aumentada (AR) se diferencia de la realidad virtual (VR) en que en la primera el usuario mantiene la sensación de presencia en el mundo real mientras en la segunda se recrea un mundo virtual total.

La realidad aumentada tiene dos funciones: aumenta la percepción de la realidad (muestra la realidad, pero elige qué se puede ver y qué no), y crea un ambiente artificial (muestra lo que no es real permitiendo ver lo imaginario), con una percepción aumentada de información útil que ayuda a la toma de decisiones.

Los sistemas de AR se caracterizan porque tienen elementos reales y virtuales en un entorno real con alto grado de interactividad, y donde se tiene la opción de registrar y posicionar la información virtual teniendo en cuenta la tridimensionalidad del mundo real [1] [3].

\section{UNITY GAME ENGINE Y VUFORIA}

El motor de videojuegos Unity es uno de los más usados para la creación de juegos interactivos en 2D, 3D y ambientes virtuales multiplataforma [5]. Unity incluye además del motor de videojuegos un ambiente integrado de desarrollo (IDE) y un framework de scripts orientado a objetos para tres lenguajes: Boo (un lenguaje de Unity similar a Python), JavaScript y C\#, permitiendo además desarrollar aplicaciones web y juegos para consolas como Xbox, Wii, y PS3, así como aplicaciones para sistemas operativos iOS, Android, Windows, Mac OS y Linux.

Otra de las ventajas de Unity es su amplio soporte técnico debido a su popularidad [12]. Popularidad que ha hecho que tanto el game engine con su IDE estén siendo empleados con mayor demanda en aplicaciones de ingeniería y medicina [2], [3], [9], [13], [16], [17].

De otra parte Vuforia es una plataforma software que permite la creación de aplicaciones de realidad aumentada para iOS y Android junto a Unity 3D [10], y trabaja como un complemento de visión por computador.

\section{METODOS}

\subsection{ARQUITECTURA}

La tarea principal de la aplicación propuesta en este artículo es usar como despliegue un dispositivo móvil con sistema operativo Android, en este caso una tableta, asistiendo en la visualización de la cirugía laparoscópica con información adicional de realidad aumentada como se muestra en la Figura 1.
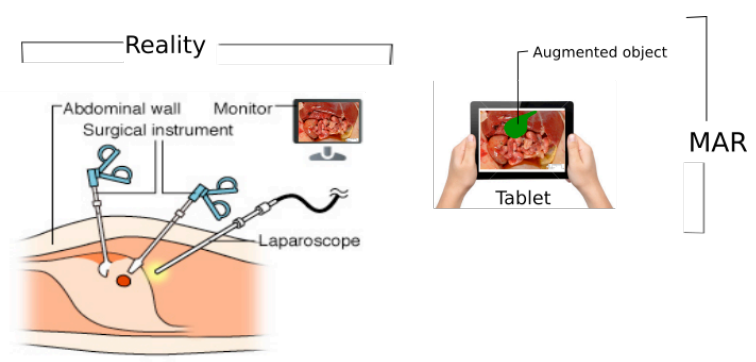

Figura 1: Esquema general del sistema propuesto

\subsection{APLICACION}

La aplicación de realidad aumentada en el dispositivo móvil (Mobile Augmented Reality - MAR) está 
compuesta por dos partes: hardware y software. El hardware incluye el dispositivo que despliega simultáneamente el ambiente real del usuario y los objetos virtuales que aumentan la realidad, en este caso una tableta con sistema operativo Android u algún tipo de HoloLens. La segunda parte la constituye el software que incluye reconocimiento y seguimiento; el cual identifica el marcador visual a partir de sus características, captura el ambiente que el usuario ve y toma la posición del marcador y la cámara. Por último, renderiza los objetos $3 \mathrm{D}$ que hacen parte de la aumentación en tiempo real y los despliega también en tiempo real. La figura 2 muestra el diagrama de la creación de la imagen.

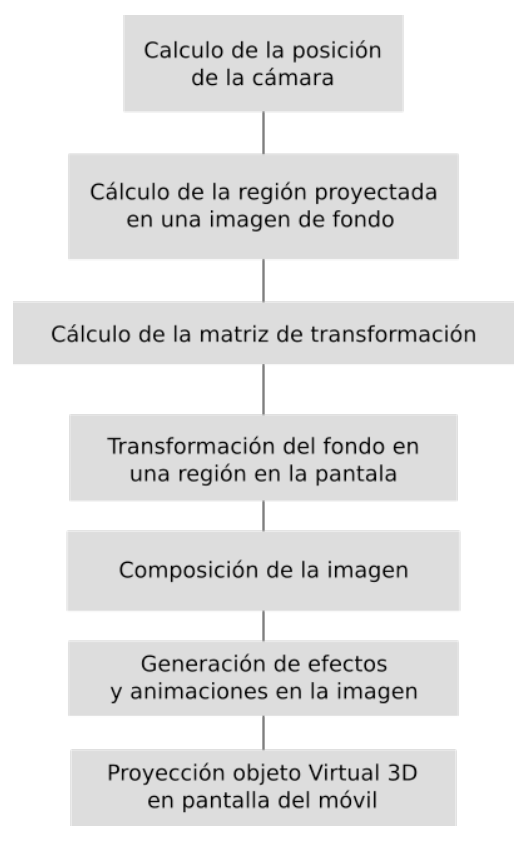

Figura 2: Diagrama de la creación de la imagen

Al importar la librería Vuforia de realidad aumentada en Unity, esta funciona como un asset (acción o complemento), y ya se pueden utilizar todos los recursos para realidad aumentada: reconocimiento de marcadores, marcadores de objetos previamente hechos, seguimiento de cámara AR, etcétera.

\section{RESULTADOS}

Para cumplir con los requerimientos de realidad aumentada en una aplicación, es necesario estimar la posición y orientación de la cámara con respecto al mundo real y viceversa. La combinación de posición $\mathrm{y}$ orientación es llamada "pose" y en este caso se utilizó la técnica de seguimiento o "tracking" de marcadores cuadrados (marker-based tracking).

\subsection{MARKER BASED AR}

Una de las áreas más trabajadas en el reconocimiento de marcadores es el reconocimiento de marcadores cuadrados (border marker o fiducial marker) en tiempo real, realizándose incluso en situaciones de difícil reconocimiento como al encontrarse el marcador girado o sesgado. Por lo tanto, este enfoque resulta ser el más popular y el primero en ser trabajado en proyectos de realidad aumentada.

Un marcador cuadrado es por lo general una imagen 2D impresa en una hoja de papel o superficie lisa. Ese tipo de marcadores son cuadrados y tienen un borde negro de tamaño visible. Durante la fase de seguimiento el sistema realiza una búsqueda de un rectángulo negro y solo si es identificado se procede a examinar el interior de la frontera para determinar el marcador real.

Dependiendo de las características del marcador se puede determinar la posición, escala y orientación con respecto a la cámara. En este caso la librería de realidad aumentada identificó marcadores cuadrados cuyas características son conocidas a partir de un proceso previo de extracción en tiempo real, y es a partir de esta identificación que se estima la posición relativa de la cámara. Este sistema está basado en un framework de seguimiento que proporciona los datos del reconocimiento y posición del marcador con respecto a la cámara, y en un game engine para la construcción del mundo virtual sobre Unity. Al desarrollar la aplicación móvil y fijar la cámara en el marcador se obtiene el despliegue en pantalla del objeto $3 \mathrm{D}$ relacionado a ese marcador. El objeto tridimensional entonces es hijo del "image target". La captura de pantalla mostrada en la figura 3 permite ver un elemento tridimensional renderizado sobre un marcador cuadrado.

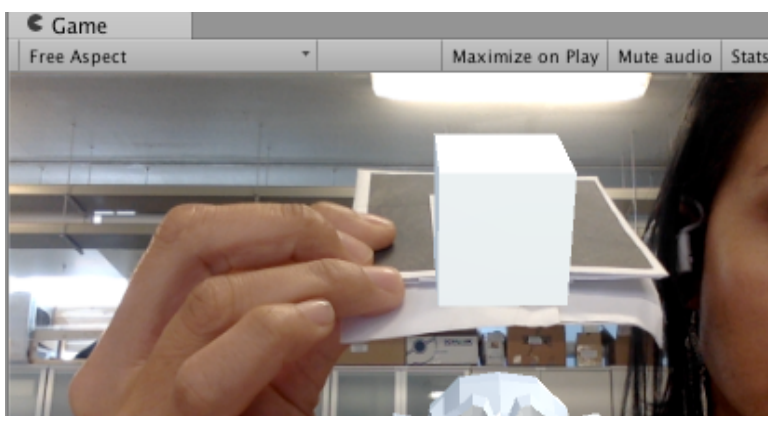

Figura 3: Objeto 3D sobre un marcador cuadrado

La prueba de deformación consistió en ubicar un objeto 3D en la realidad mixta con características plásticas, es decir que ante presencia de una fuerza de contacto su morfología se afecta, y en la no presencia de dicha fuerza el objeto recupera su forma. Es de aclarar que debido a que solo es una prueba se utilizó un modelo 3D previamente hecho, en este caso el 
modelo típico de Blender en forma de cabeza de mono. Ese modelo 3D se importó en el proyecto de Unity ubicándolo en un lugar central dentro de la imagen de la cámara de realidad aumentada. Se trabajó también con otro objeto $3 \mathrm{D}$, un cubo, usando la herramienta de Unity para creación de objetos 3D. Dicho cubo se asoció al marcador de AR. Se crearon los scripts necesarios para que al ser movido el marcador físico el desplazamiento del cubo fuera similar, y al ser tocada la cabeza del mono (objeto 3D importado de Blender) con el cubo, esta se deformara como se observa en figura 4.

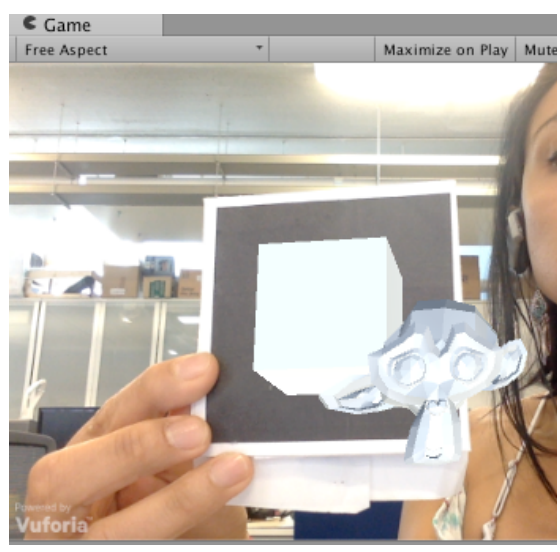

Figura 4. Prueba de deformación entre objetos en el entorno de realidad aumentada

\subsection{OBJETO AR CON INTERACCIONES DEFORMANTES}

La deformación simulada de la vesícula como una superficie deformable en Unity, constituyó la segunda prueba que involucró la construcción de una superficie de vértices deformables de manera individual, la cual se creó a partir de una red de vértices y una superficie de conexión entre ellos. Es importante tener una buena cantidad de vértices no solo para presentar una mejor apariencia en el objeto tridimensional, sino también porque permitirá una deformación más natural del objeto.

El objeto deformable (vesícula) fue realizado con Blender y luego importado dentro de Unity. La vesícula se convierte en una superficie deformable realizando un script que modifica el comportamiento de los vértices de acuerdo a la interacción con otro objeto 3D. La primera acción es acceder a la malla de vértices y copiar las posiciones iniciales de ellos sin deformación, guardando aquellos valores en un vector. Para simular el comportamiento de los vértices y de la superficie en su totalidad, se tuvo como referencia la posición original de los vértices del objeto sin deformación y el actual desplazamiento de los vértices modificados por la interacción.
Los vértices se mueven con cierta velocidad (dependiendo de la fuerza de contacto), y por lo tanto se debe obtener ese valor en cada momento. Para controlar cómo la malla es deformada se usa una entrada interactiva (ya sea el toque con otro objeto o un toque en pantalla como se describirá más adelante), relacionando este toque con una fuerza que modifica la posición y velocidad de los vértices.

Otro cuerpo 3D (cubo) se asoció con el marcador AR. En cuanto ese otro cuerpo está en contacto con la superficie deformable, los vértices de este se mueven con una fuerza inicial que afecta fuertemente a los vértices más cercanos al punto de toque, y en menor proporción a los más alejados, como se observó en la figura 4. Cada vértice afectado retorna a su posición inicial con una fuerza restaurativa y un coeficiente de amortiguamiento, una vez el efecto deformador desaparece.

De las librerías físicas de Unity solo se usó la función llamada mesh collider para detectar la zona de contacto, en tanto que el comportamiento de los vértices se generó con el script codificado para el objeto deformable.

Otro script es asociado con el cuerpo del marcador AR y contiene la detección del toque con el objeto 3D (cubo), y las constantes iniciales para el cálculo de la fuerza.

Posteriormente una vesícula virtual fue relacionada a un nuevo marcador sobre un hígado sintético en el mundo real. Se realizó el ejecutable de la aplicación móvil (apk) y se instaló en la tableta. La tableta Samsumg tab2 fue ubicada en un robot y la cámara alineada con la vista del hígado sintético. Dicho montaje se realizó en el laboratorio del grupo nBIO (Grupo de investigación en Neuroingeniería Biomédica) de la Universidad Miguel Hernández, en Elche, España, sobre un robot de asistencia quirúrgica actuando sobre una caja de entrenamiento laparoscópico como se puede ver en la Figura 5.

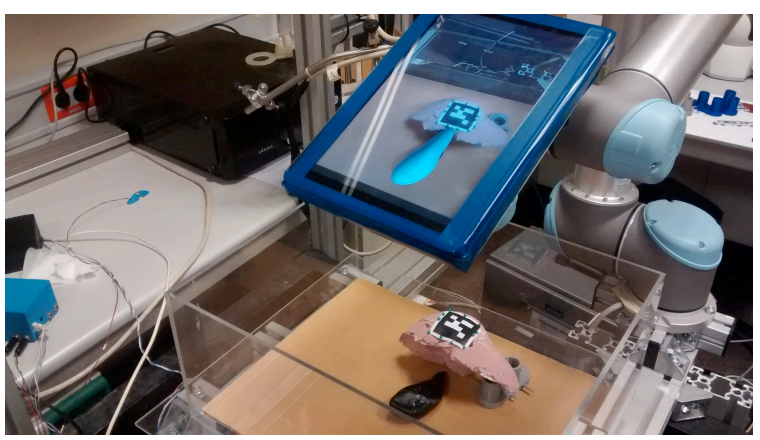

Figura 5: Fotografía de montaje con el dispositivo de despliegue y marcador físico ubicado en el hígado sintético 
Con el fin de probar la deformación de la superficie en el script de detección de colisión, se incluyó una funcionalidad para simular la deformación de la vesícula ante un toque en la pantalla de la tableta, tal como se puede observar en la figura 6 , de tal manera que cuando se toque la pantalla del móvil la vesícula virtual sufra deformación.

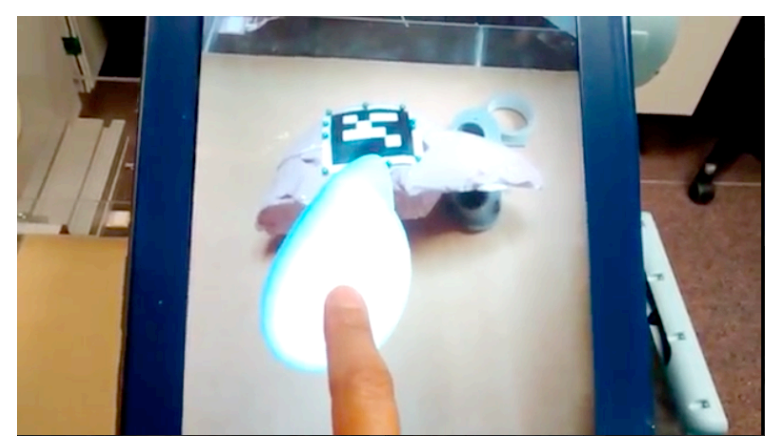

Figura 6: Toque de la pantalla y deformación de la vesícula virtual

\section{CONCLUSIONES}

La realidad aumentada es una técnica que puede ser usada como herramienta para facilitar el posicionamiento visual de cirujanos dentro de operaciones laparoscópicas, permitiendo sumar información creada de forma artificial, ubicándola dentro del campo visual que es proporcionado por la cámara endoscópica. Este tipo de trabajos permiten una representación más intuitiva del campo de trabajo al cual se enfrenta el cirujano.

En este trabajo se realizó una prueba estándar con marcadores cuadrados también llamados "border markers", usando la librería Vuforia de realidad aumentada y como base el entorno integrado de desarrollo (IDE) de Unity, así como su motor multiplataforma de videojuegos.

Como resultado se tiene que la elección de Vuforia como librería de reconocimiento de marcadores para realidad aumentada, y de Unity como IDE de desarrollo, resulta ser satisfactoria para marcadores cuadrados. El seguimiento del marcador es rápido y la aplicación para el sistema operativo Android resulta ser muy estable. Uno de los mayores obstáculos en el uso de esta librería radica en la exigencia de extracción previa de las características del marcador, la cual se realiza con una aplicación web proporcionada por los fabricantes de Vuforia y que es llamada "target manager". Dicha aplicación entrega una base de datos de las características del marcador, la cual es importada en Unity y que sin duda proporciona un reconocimiento rápido de él. Sin embargo, se evidencia la necesidad de usar otra librería de visión que permita mayor flexibilidad en el manejo de los algoritmos para la identificación y seguimiento de marcadores naturales. En cuanto al IDE de desarrollo, el resultado de adicionar interacciones físicas en los objetos aumentados resulta ser totalmente posible y estable.

Para trabajos futuros se plantea el uso del IDE de Unity con el complemento de OpenCV para Unity, debido principalmente a la necesidad de implementar mejores técnicas en los algoritmos de extracción de características y esquemas de seguimiento basados en aprendizaje de máquina, que permitan una mejor ubicación de los elementos virtuales en la realidad mixta. De esta manera se ampliará el trabajo a marcadores naturales.

\section{Agradecimientos}

"C-HALS" - Referencia: DPI2013-47196-C3-2-R [AEI/FEDER, UE], financiado por la Agencia Estatal de Investigación (AEI) y por la Unión Europea a través del Fondo Europeo de Desarrollo Regional FEDER - "Una manera de hacer Europa"

\section{Referencias}

[1] Autorino, R., Cadeddu, J. A., Desai, M. M., Gettman, M., Gill, I. S., Kavoussi, L. R., ... \& Kaouk, J. H. (2011). Laparoendoscopic singlesite and natural orifice transluminal endoscopic surgery in urology: a critical analysis of the literature. European Urology, 59(1), pp. 26-45.

[2] Bae, J. H. (2016). Development of smart game based on multi-platform game engine. International Journal of Multimedia and Ubiquitous Engineering, 11(3), pp. 345350.

[3] Cristie, V., Berger, M., Bus, P., Kumar, A., \& Klein, B. (2015). CityHeat: visualizing cellular automata-based traffic heat in Unity3D. SIGGRAPH Asia 2015 Visualization in High Performance Computing, p. 6. ACM.

[4] Ćuković, S., Gattullo, M., Pankratz, F., Devedžić, G., Carrabba, E., \& Baizid, K. (2016). Marker based vs. natural feature tracking augmented reality visualization of the 3D foot phantom. Electrical and Bio-medical Engineering, Clean Energy and Green Computing, 1(1), p. 8.

[5] Fürst, J., Fierro, G., Bonnet, P., \& Culler, D. E. (2014). BUSICO 3D: building simulation and control in unity 3D. 12th ACM Conference on Embedded Network Sensor Systems, pp. 326327. 
[6] Kipper, G., \& Rampolla, J. (2012). Augmented Reality: an emerging technologies guide to AR. Elsevier.

[7] Marescaux, J., \& Diana, M. (2015). Next step in minimally invasive surgery: hybrid imageguided surgery. Journal of pediatric surgery, 50(1), pp. 30-36.

[8] Moreno, M. R., Moraes, T. F., Amorim, P. H., da Silva, J. V. L., \& Rodriguez, C. A. (2012). Virtual open source environment for training and simulation of laparoscopic surgery. In XII Work-shop de Informática Médica (WIM2012)- XXXII Congresso da Sociedade Brasileira de Computação, pp. 1-4.

[9] Narahara, T., Abbruzzese, K. M., \& Foulds, R. A. (2015). Haptic collaboration: biomedical engineering meets digital design. SIGGRAPH 2015, p. 20. ACM.

[10] Peng, H. (2015). Application Research on Face Detection Technology based on OpenCV in Mobile Augmented Reality. International Journal of Signal Processing, Image Processing and Pattern Recognition, 8(4), pp. 249-256.

[11] Rané, A., Rao, P., \& Rao, P. (2008). Singleport-access nephrectomy and other laparoscopic urologic procedures using a novel laparoscopic port (R-port). Urology, 72(2), pp. 260-263.

[12] Simonetti Ibañez, A., \& Paredes Figueras, J. (2013). Vuforia v1. 5 SDK: Analysis and evaluation of capabilities (Master's thesis, Universitat Politècnica de Catalunya, España).

[13] Soto, C., Vargas, M., Uribe-Quevedo, A., Jaimes, N., \& Kapralos, B. (2015). A stereoscopic $3 \mathrm{~d}$ human eye examination app. International Conference on Interactive Mobile Communication Technologies and Learning (IMCL), pp. 236-238.

[14] Van der Pas, M. H., Haglind, E., Cuesta, M. A., Fürst, A., Lacy, A. M., Hop, W. C., \& Bonjer, H. J. (2013). COlorectal cancer Laparoscopic or Open Resection II (COLOR II) Study Group Laparoscopic versus open surgery for rectal cancer (COLOR II): short-term outcomes of a randomised, phase 3 trial. Lancet Oncol, 14(3), pp. 210-8.

[15] Weizman, N. F., Maurer, R. M. A., Einarsson, J. I., Vitonis, A. F., \& Cohen, S. L. (2014). Survey on Barriers to Adoption of Laparoscopic Surgery. Journal of Minimally Invasive Gynecology, 21(6), p. S46.

[16] Woo, J. H., Peterson, M. A., \& Gleason, B. (2016). Developing a Virtual Campus Model in an Interactive Game-Engine Environment for Building Energy Benchmarking. Journal of Computing in Civil Engineering,30(5), p.C4016005.

[17] Zhong, H., \& Xiao, J. (2015). Apply technology acceptance model with big data analytics and unity game engine. 6th IEEE International
Conference on Software Engineering and Service Science (ICSESS), pp. 19-24. 